\title{
Two-year results of a multicenter study of the ab interno gelatin implant in medically uncontrolled primary open-angle glaucoma
}

\author{
Herbert Reitsamer ${ }^{1} \cdot$ Chelvin Sng $^{2,3,4} \cdot$ Vanessa Vera $^{5} \cdot$ Markus Lenzhofer $^{1} \cdot$ Keith Barton $^{2,3} \cdot$ Ingeborg Stalmans ${ }^{6} \cdot$ For \\ The Apex Study Group
}

Received: 9 October 2018 / Revised: 9 January 2019 / Accepted: 11 January 2019 / Published online: 13 February 2019

(C) The Author(s) 2019

\begin{abstract}
Purpose To evaluate the effectiveness of an ab interno subconjunctival gelatin implant as primary surgical intervention in reducing intraocular pressure (IOP) and IOP-lowering medication count in medically uncontrolled moderate primary openangle glaucoma (POAG).

Methods In this prospective, non-randomized, open-label, multicenter, 2-year study, eyes with medicated baseline IOP 18$33 \mathrm{mmHg}$ on 1-4 topical medications were implanted with (phaco + implant) or without (implant alone) phacoemulsification. Changes in mean IOP and medication count at months 12 (primary outcomes) and 24, clinical success rate (eyes [\%] achieving $\geq 20 \%$ IOP reduction from baseline on the same or fewer medications without glaucoma-related secondary surgical intervention), intraoperative complications, and postoperative adverse events were assessed.

Results The modified intent-to-treat population included 202 eyes (of 218 implanted). Changes (standard deviation) in mean IOP and medication count from baseline were -6.5 (5.3) $\mathrm{mmHg}$ and $-1.7(1.3)$ at month 12 and $-6.2(4.9) \mathrm{mmHg}$ and $-1.5(1.4)$ at month 24, respectively (all $P<0.001)$. Mean medicated baseline IOP was reduced from 21.4 (3.6) to $14.9(4.5) \mathrm{mmHg}$ at 12 months and $15.2(4.2) \mathrm{mmHg}$ at 24 months, with similar results in both treatment groups. The clinical success rate was $67.6 \%$ at 12 months and $65.8 \%$ at 24 months. Overall, 51.1 (12 months) and 44.7\% (24 months) of eyes were medication-free. The implant safety profile compared favorably with that published for trabeculectomy and tube shunts.

Conclusions The gelatin implant effectively reduced IOP and medication needs over 2 years in POAG uncontrolled medically, with an acceptable safety profile.

ClinicalTrials.gov registration number: NCT02006693 (registered in the USA).
\end{abstract}

Keywords Glaucoma $\cdot$ Stent $\cdot$ Implant $\cdot$ Minimally invasive glaucoma surgery $\cdot$ Ab interno $\cdot$ XEN

Results from interim analyses were presented in part at: American Glaucoma Society 2016 Annual Meeting, March 3-6, 2016, Fort Lauderdale, FL, USA; American Society of Cataract and Refractive Surgery (ASCRS) Symposium and Congress, May 6-10, 2016, New Orleans, LA, USA; European Association for Vision and Eye Research Conference, October 5-8, 2016, Nice, France; and 12th European Glaucoma Society Congress, June 19-22, 2016, Prague, Czech Republic.

Chelvin Sng

chelvin@gmail.com

1 Department of Ophthalmology and Optometry, University Clinic Salzburg, SALK/Paracelsus Medical University, Salzburg, Austria

2 Moorfields Eye Hospital, London, UK

3 Department of Ophthalmology, National University Hospital, 1E Kent Ridge Road, NUHS Tower Block, Level 7, Singapore 119228, Singapore
4 Singapore Eye Research Institute, Singapore, Singapore

5 Department of Ophthalmology, Unidad Oftalmologica de Caracas, Caracas, Venezuela

6 Department of Ophthalmology, University Hospitals UZ Leuven, Leuven, Belgium 


\section{Introduction}

Topical therapy is the usual first-line treatment for open-angle glaucoma, but is often hampered by poor adherence $[1,2]$. Traditional subconjunctival drainage procedures, such as trabeculectomy and tube shunts, lower IOP most effectively but are relatively invasive and associated with both short- and longer-term complications that may result in significant loss of visual acuity [3-7]. Newer minimally invasive glaucoma surgery (MIGS) that permits earlier intervention is becoming part of the treatment armamentarium for glaucoma, providing a better safety profile than conventional approaches [8]. MIGS devices that can be implanted in conjunction with cataract surgery to facilitate aqueous drainage into Schlemm's canal [9] or the supraciliary space [10] offer modest IOP lowering. An ab interno gelatin stent $(\mathrm{XEN} ® 45$, Allergan plc, Dublin, Ireland) that also meets the criteria for MIGS [11] bypasses conventional outflow pathways that are known to be obstructed in primary open-angle glaucoma (POAG) by creating a connection between the anterior chamber and subconjunctival space [8], in a manner similar to the gold standard trabeculectomy. The device is implanted ab interno, either as a stand-alone procedure or in combination with cataract surgery, without conjunctival dissection. The hydrophilic gelatin implant swells and conforms to surrounding tissues, which helps maintain its position post-implantation.

Results from studies demonstrating the IOP-lowering performance and safety of the gelatin implant at 1 year across a spectrum of glaucoma patients have been published [12-17]. The present study was designed to evaluate, over 2 years in typical clinical settings, the effectiveness of the gelatin implant as primary surgical intervention in reducing IOP and the number of topical IOP-lowering medications in patients with POAG uncontrolled on topical therapy.

\section{Methods}

\section{Study design}

This prospective, non-randomized, open-label, multicenter clinical study (ClinicalTrials.gov identifier: NCT02006693) was conducted between December 2013 and January 2017 in eight countries (Austria, Belgium, England, Germany, Italy, Poland, Spain, and Switzerland). The study complied with Good Clinical Practice/International Council for Harmonisation Guidelines, the Declaration of Helsinki, and all applicable country-specific regulations governing the conduct of clinical research, depending on which provided greater protection to the individual. The protocol was approved by an independent ethics committee prior to study start, and all patients were to provide written informed consent before initiating treatment.

\section{Study population}

The inclusion criteria were as follows: diagnosis of moderate POAG (defined by a mean deviation score between -3 and $-12 \mathrm{~dB}$ ) uncontrolled on topical therapy; medicated IOP $\geq 18$ and $\leq 33 \mathrm{mmHg}$; use of one to four topical IOP-lowering medications; area of healthy, free, and mobile conjunctiva in the target quadrant; Shaffer angle grade $\geq 3$ in the target quadrant; $\geq 18$ years of age; signed written informed consent; and availability, willingness, and sufficient cognitive awareness to comply with the examination procedures and schedule.

Exclusion criteria included a diagnosis of any glaucoma other than POAG; prior incisional glaucoma surgery (prior iridotomy was acceptable if angles were open); prior cataract surgery in the study eye $\leq 3$ months before study treatment; presence of scarring, prior surgery, or other pathologies in the conjunctiva (target quadrant); history of corneal surgery/ disease; central corneal thickness $\leq 490$ or $\geq 620 \mu \mathrm{m}$; presence of vitreous in the anterior chamber; presence of intraocular silicone oil; clinically significant inflammation or infection in the study eye within 30 days prior to the preoperative visit; active ophthalmic disease/disorder that could confound study results; impaired episcleral venous drainage; and known or suspected allergy/sensitivity to drugs required for the implantation (including anesthesia), or any of the device components (e.g., bovine or porcine products, and glutaraldehyde).

Both eyes could be implanted (study eyes) provided they met the eligibility criteria and surgeries for each eye were performed at least 30 days apart.

\section{Perioperative procedures}

The gelatin implant was placed ab interno either as a standalone procedure (implant alone) or in combination with cataract surgery (phaco + implant), based on whether the surgeon and patient deemed cataract surgery necessary at the time of glaucoma surgery.

Consistent with typical clinical practice, investigators could adjust the preoperative medication regimen as believed necessary/appropriate. Recommendations included a topical steroid (prednisolone acetate $1 \%$ or equivalent, or benzalkonium chloride [BAK]-free difluprednate $0.05 \%$ ) four times daily (QID) in the study eye one week before surgery, and a topical antibiotic (fluoroquinolone or equivalent, preferably BAK-free) QID on day - 1 (preoperative). Topical (in the study eye) or systemic IOP-lowering medications were to be suspended on day 0 (surgery day). The surgery was performed using standard ophthalmic operating techniques and perioperative medications (including anesthesia), as customary for the investigator. Adjunctive antifibrotic therapy was administered pre-/perioperatively via subconjunctival injection, at the surgeon's discretion (including type and dose). 
In the implant alone group, an ab interno approach (described by Vera and Horvath [8]) was used to place the gelatin stent, connecting the anterior chamber to the subconjunctival space. General surgical steps for implantation included creating temporal clear corneal main and side port incisions; filling the anterior chamber with cohesive viscoelastic; inserting the needle tip of the injector through the main incision and advancing across the anterior chamber (toward the superior-nasal quadrant), with needle entry at the desired angle position and advancement through the sclera using a second instrument at the side port to provide stabilization and counterforce; visualizing the needle and needle tip bevel in the subconjunctival space; deploying the gelatin stent; removing the injector and viscoelastic; pressurizing the anterior chamber; and creating a subconjunctival bleb with a balanced salt solution. All incisions were hydrated at the end of the surgery. The target for an ideally positioned stent was $1 \mathrm{~mm}$ in the anterior chamber, $2 \mathrm{~mm}$ in the scleral tunnel, and $3 \mathrm{~mm}$ in the subconjunctival space. If incorrectly positioned, the device could be adjusted or exchanged.

In the phaco + implant group, phacoemulsification was performed and an intraocular lens was inserted, followed by placement of the gelatin implant if the cataract surgery was successful and uncomplicated. If complications that could potentially impact the study results (such as corneal burn, vitreous loss requiring vitrectomy, and placement of an anterior chamber lens) occurred during cataract surgery, the eye was withdrawn from the study.

The postoperative treatment regimen was per investigator's discretion. Recommendations included topical antibiotic (fluoroquinolone or equivalent, preferably BAKfree) QID for 1 week, as well as topical steroid (prednisolone acetate $1 \%$ or equivalent, or BAK-free difluprednate $0.05 \%$ ) QID for $\leq 4$ weeks and titrated thereafter based on clinical assessment of postoperative inflammation. If a patient required further IOP lowering postoperatively, the investigator had the option of reintroducing ocular hypotensive medications in a stepwise fashion (i.e., 1 drug class at a time) and/or needling the bleb. Consistent with the American Academy of Ophthalmology's Preferred Practice Pattern Guidelines [1], needling was part of the standard postoperative care to improve aqueous flow and lower IOP based on the investigator's clinical assessment of bleb function. Consistent with other recent studies [18, 19], needling was not considered an adverse event (AE) or glaucomarelated secondary surgical intervention (SSI) but was documented as a postoperative procedure; it could be performed at any point in the postoperative period, as believed necessary by the investigator. No specific protocol was mandated, and use of an antifibrotic agent at the time of needling was also left to the investigator's discretion.

\section{Assessments}

Postoperative visits were scheduled at day 1, weeks 1 and 2, and months $1,3,6,9,12,18$, and 24 . IOP was determined at medicated baseline and each postoperative visit using Goldmann applanation tonometry and a masked, two-person method [20]; two consecutive measurements were taken, followed by a third if the first two differed by $\geq 3 \mathrm{mmHg}$. The average or median IOP was used for analysis, depending on whether two or three measurements were taken, respectively. Use of topical IOP-lowering medications was assessed at baseline and all postoperative visits.

Safety assessments included intraoperative complications (day 0 only), monocular best-corrected visual acuitymeasured in Snellen (at all postoperative visits except day 1) and converted into logMAR for analysis, slit-lamp biomicroscopy, and postoperative AEs (at each postoperative visit). AEs of interest, such as shallow anterior chamber with iridocorneal touch, choroidal effusion, macular edema, macular folds, corneal erosion, and corneal edema, were specifically assessed and documented. Ophthalmoscopy (cup/disc ratio), pachymetry (central corneal thickness), and visual field (mean deviation) were assessed at baseline, month 12, and month 24 .

\section{Outcomes and analyses}

All effectiveness analyses were performed using the modified intent-to-treat (mITT) population (i.e., all enrolled eyes [with verified informed consent documentation] that received an implant and met the IOP and IOP-lowering medication count inclusion criteria). The primary effectiveness outcomes were the changes in mean IOP and mean number of topical IOPlowering medications in the study eyes from baseline to month 12; these parameters were also assessed at all other postoperative visits up to 24 months (secondary effectiveness outcomes).

Clinical success was defined as achieving $\geq 20 \%$ IOP reduction on the same or fewer IOP-lowering medications at month 12 (or 24), compared with baseline, without glaucoma-related SSI (which did not include needling) or intention to be converted to another procedure during the study.

Other effectiveness outcomes included the mean IOP and mean IOP-lowering medication count (topical) at each study visit, as well as the proportion of eyes achieving specific target IOPs, proportion of topical medication-free eyes and their mean IOP, proportion of eyes requiring needling, along with the mean number of needling procedures per eye, number of eyes with 1,2,3, or $>3$ needling procedures, overall needling rate, needling rate by site, and clinical success rate in needled eyes, at 12 and 24 months. The median needling rate was also calculated based on the month-24 needling rate for each site. AEs were summarized by counts and percentages, using the 
safety population (i.e., all eyes enrolled in the study that received the gelatin implant). Descriptive statistics were used to summarize all endpoints in the overall population, based on observed data (i.e., without imputation for missing data). Statistical testing was also performed to compare the changes from baseline in mean IOP and mean IOP-lowering medication count at months 12 and 24 between treatment groups. Because 19 patients had both eyes treated, a random effect model [21] was used to adjust for correlation between those eyes. Analysis was also performed using only one eye per patient (i.e., the first treated eye). In addition, the differences in needling procedures per eye between groups were analyzed using the modified Wilcoxon rank-sum test (adjusting for correlation [22]). All analyses were generated using the SAS® software version 9.3 (SAS Institute Inc., Cary, NC, USA).

Enrollment of up to 200 eyes was planned; due to unknown variability of the procedure's effect on IOP, a formal calculation of sample size was not performed. The study was remotely monitored using a risk-based monitoring approach with one onsite visit at the end of the study. Although an interim analysis at 12 months was performed as planned and interim data cuts presented in scientific meetings (listed above), reported herein is the final analysis, performed after completion of the 24-month visit.

\section{Results}

\section{Demographics, baseline characteristics, and surgical parameters}

Overall, 240 eyes (217 patients) entered the study and 219 (200 patients) were enrolled (Fig. 1). A total of 218 eyes of 199 patients received the gelatin implant at 21 sites and were included in the safety population. The number (\%) of patients implanted at each site ranged from $1(0.5)$ to 28 (14.1) (mean 9.5; median 7.0). Overall, 197/218 (90.4\%) eyes completed the 12-month visit; 174/218 (79.8\%) completed the 24-month visit, while 44/218 (20.2\%) discontinued the study (Fig. 1). No eye was withdrawn due to complications from cataract surgery. Data were comparable in the mITT population, with $182 / 202(90.1 \%)$ and $161 / 202(79.7 \%)$ eyes completing the
Fig. 1 Patient disposition. mITT, modified intent-to-treat; Phaco, phacoemulsification with intraocular lens placement. ${ }^{\text {a }}$ Patients/eyes with verified informed consent documentation (incomplete consent documentation, $N=21){ }^{\mathrm{b}}$ Enrolled and received an implant (did not receive an implant, $N=$ 1). ${ }^{\mathrm{c}}$ All enrolled eyes that received an implant and met the IOP and IOP-lowering medication count inclusion criteria. ${ }^{\mathrm{d}}$ No specific reasons were recorded

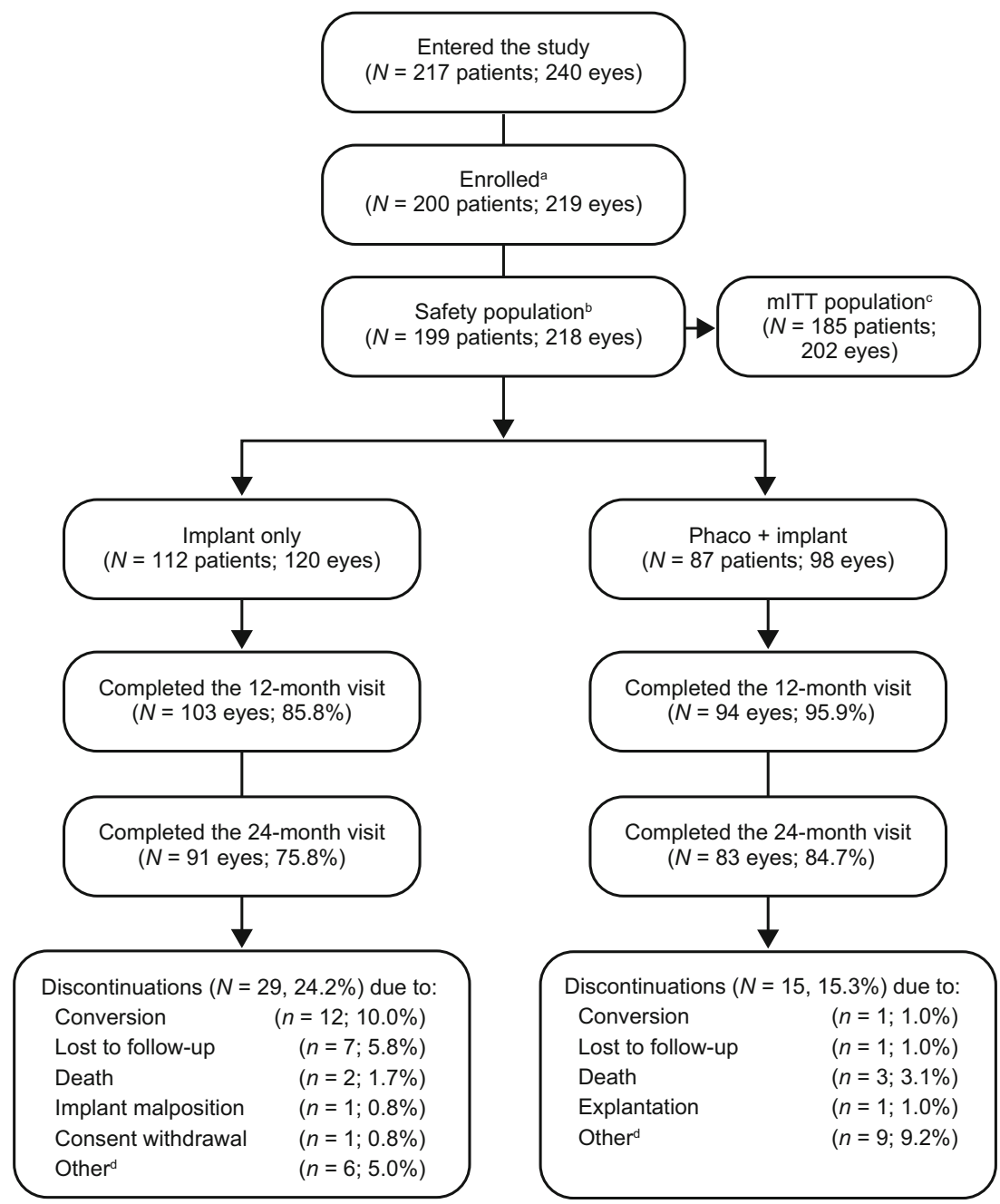


12- and 24-month visits, respectively, and 41/202 (20.3\%) discontinuing the study. Patient demographics and baseline characteristics for the mITT population are summarized in Table 1; a total of 25 (23.6\%) eyes in the implant alone group were pseudophakic.

Adjunctive antifibrotic therapy (administered by subconjunctival injection) was used in all eyes; $99 \%$ received mitomycin $\mathrm{C}$ (MMC), while the remaining $1 \%$ received 5fluorouracil (5-FU). The time of administration and absolute doses are detailed in Table 2.

\section{Effectiveness}

In the mITT population $(N=202)$, the change standard deviation (SD) in mean IOP from preoperative medicated baseline at 12 months (primary time point) was $-6.5(5.3) \mathrm{mmHg}$ $(P<0.001)$. The change (SD) in mean number of IOPlowering medications was $-1.7(1.3)(P<0.001)$. Notably, the changes in both outcomes were also statistically significant at all other post-operative visits $(P<0.001)$, reaching 6.2 (4.9) $\mathrm{mmHg}$ and -1.5 (1.4) at month 24 , respectively (Fig. 2). The mean percentage change in IOP from medicated baseline was $-29.3 \%$ at month 12 and $-27.8 \%$ at month 24 (Fig. 2).
Overall, results were similar in both treatment arms. The mean changes in IOP from medicated baseline were -6.6 (5.6) and - 6.4 (5.0) mmHg at month 12 and - 6.4 (5.2) and -5.9 (4.6) $\mathrm{mmHg}$ at month 24 in the implant alone and phaco + implant groups, respectively $(P>0.50)$. In these groups, the mean changes in IOP-lowering medication count were $-1.8(1.3)$ and $-1.6(1.2)$ at month 12 and $-1.5(1.5)$ and $-1.5(1.2)$ at month 24 , respectively $(P>0.48)$. The mean percentage changes in IOP from medicated baseline were -29.6 (month 12) and $-28.2 \%$ (month 24 ) in the former group and -29.1 (month 12 ) and $-27.2 \%$ (month 24 ) in the latter (Fig. 2).

At 24 months, outcomes were also similar in pseudophakic eyes that received the implant alone (IOP reduction, $-8.4 \mathrm{mmHg}$; reduction in medication number, $-1.5 ; n=15$ in the mITT population) versus the overall implant alone group and the phaco + implant group. The outcomes also appeared similar between phakic $(n=80)$ and pseudophakic $(n=25)$ eyes that received the implant alone, although no statistical comparisons were made between these groups.

Because 19 patients had both eyes implanted, two sensitivity analyses were conducted. One used a random effect model to adjust for correlation between those eyes [21], while the other included only one eye per patient. Results of both
Table 1 Patient demographics and baseline characteristics (mITT population)

\begin{tabular}{|c|c|c|c|}
\hline Demographics/characteristics & $\begin{array}{l}\text { Implant alone } \\
N=106\end{array}$ & $\begin{array}{l}\text { Phaco + implant } \\
N=79\end{array}$ & $\begin{array}{l}\text { Total } \\
N=185\end{array}$ \\
\hline Mean age, years (SD) & $68.3(11.7)$ & $76.5(6.1)$ & $71.8(10.5)$ \\
\hline \multicolumn{4}{|l|}{ Sex, $n(\%)$} \\
\hline Female & $55(51.9)$ & $40(50.6)$ & $95(51.4)$ \\
\hline Male & $51(48.1)$ & $39(49.4)$ & $90(48.6)$ \\
\hline \multicolumn{4}{|l|}{ Race, $n(\%)$} \\
\hline White & $103(97.2)$ & $75(94.9)$ & $178(96.2)$ \\
\hline Black/African-American & $2(1.9)$ & $1(1.3)$ & $3(1.6)$ \\
\hline Asian & $1(0.9)$ & $3(3.8)$ & $4(2.2)$ \\
\hline Preoperative IOP, $\mathrm{mmHg}(\mathrm{SD})^{\mathrm{a}}$ & $21.7(3.8)$ & $21.0(3.4)$ & $21.4(3.6)$ \\
\hline Mean IOP-lowering medications, $n(\mathrm{SD})^{\mathrm{a}}$ & $2.7(0.9)$ & $2.5(0.9)$ & $2.7(0.9)$ \\
\hline \multicolumn{4}{|l|}{ Use of IOP-lowering agents, $n(\%)^{\mathrm{a}, \mathrm{b}}$} \\
\hline$\beta$-Blockers & $94(82.5)$ & $66(75.0)$ & $160(79.2)$ \\
\hline Carbonic anhydrase inhibitors & $82(71.9)$ & $51(58.0)$ & $133(65.8)$ \\
\hline Parasympathomimetics & $1(0.9)$ & $3(3.4)$ & $4(2.0)$ \\
\hline Prostaglandin analogs & $102(89.5)$ & $83(94.3)$ & $185(91.6)$ \\
\hline Sympathomimetics & $40(35.1)$ & $26(29.5)$ & $66(32.7)$ \\
\hline Pseudophakic, $n(\%)$ & $25(23.6)^{\mathrm{c}}$ & 0 & $25(13.5)$ \\
\hline Average visual field mean deviation, $\mathrm{dB}(\mathrm{SD})^{\mathrm{a}}$ & $-7.9(8.6)$ & $-8.0(9.2)$ & $-8.0(8.9)$ \\
\hline
\end{tabular}

IOP, intraocular pressure; mITT, modified intent-to-treat; phaco, phacoemulsification with intraocular lens placement; SD, standard deviation

${ }^{\mathrm{a}}$ Based on the number of eyes in the implant alone group $(N=114)$, the phaco + implant group $(N=88)$, and the total population $(N=202)$

${ }^{\mathrm{b}}$ Totals exceed $100 \%$ in each cell because several patients/eyes were using multiple IOP-lowering agents

${ }^{\mathrm{c}}$ The remaining eyes were phakic $(n=80)$ or aphakic $(n=1)$ 
Table 2 Time and absolute dose of adjunctive antifibrotic therapy administered during surgery (mITT population)

\begin{tabular}{llll}
\hline & $\begin{array}{l}\text { Implant alone } \\
(N=114)\end{array}$ & $\begin{array}{l}\text { Phaco + implant } \\
(N=88)\end{array}$ & $\begin{array}{l}\text { Total } \\
(N=202)\end{array}$ \\
\hline $\begin{array}{ll}\text { Absolute dose }(\mu \mathrm{g}) \\
10, n(\%)^{\mathrm{a}}\end{array}$ & $85(74.6)$ & $72(81.8)$ & $157(77.7)$ \\
$20, n(\%)^{\mathrm{a}}$ & $21(18.4)$ & $7(8.0)$ & $28(13.9)$ \\
$>20-40, n(\%)^{\mathrm{a}}$ & $7(6.1)$ & $4(4.5)$ & $11(5.4)$ \\
$60-80, n(\%)^{\mathrm{a}}$ & $1(0.9)$ & $3(3.4)$ & $4(2.0)$ \\
$500, n(\%)^{\mathrm{b}}$ & 0 & $2(2.3)$ & $2(1.0)$ \\
Time of administration & & & $15(7.4)$ \\
Day before surgery & $9(7.9)$ & $6(6.8)$ & $169(83.7)$ \\
Before implantation & $100(87.7)$ & $69(78.4)$ & $4(4.5)$ \\
After implantation & 0 & $4(4.5)$ & $14(6.9)$ \\
Unspecified (perioperative) & $5(4.4)$ & $9(10.2)$ & \\
\hline
\end{tabular}

mITT, modified intent-to-treat; phaco, phacoemulsification with intraocular lens placement

${ }^{\text {a }}$ Mitomycin C

b 5-Fluorouracil sensitivity analyses were similar to the effectiveness outcomes described in the above paragraphs.

The clinical success rate was $67.6 \%$ at 12 months and $65.8 \%$ at 24 months in the mITT population (Figs. 3 and 4). Looking at specific IOP targets achieved at 12 and 24 months, $55.6(n=99 / 178)$ and $48.4 \%(n=78 / 161)$ of eyes with available data achieved IOP reductions $\geq 30 \%$ from preoperative medicated baseline at 12 and 24 months, respectively (Fig. 5). In addition, the proportion of eyes achieving IOP $\leq 18, \leq 15$, and $\leq 12 \mathrm{mmHg}$ was 83.7, 60.7, and $27.5 \%$ at 12 months, and 85.1, 62.7, and $24.2 \%$ at 24 months, respectively (Fig. 6).

Remarkably, $51.1(n=91 / 178)$ and $44.7 \%(n=72 / 161)$ of eyes with available data at 12 and 24 months were medicationfree (topical), with a mean IOP (SD) of 13.8 (3.1) and 14.3 (3.1) $\mathrm{mmHg}$, respectively (Table 3 ). Among the medicationfree eyes, $79 / 91(86.8 \%)$ and 59/72 (81.9\%) achieved clinical success at 12 and 24 months, respectively.

\section{Needling}

In the mITT population, the overall needling rate was $41.1 \%$ ( $n=83 / 202$ ), without statistically significant differences between the implant alone $(43.9 \% ; n=50 / 114$ eyes) and phaco + implant $(37.5 \% ; n=33 / 88)$ groups at month $24(P>0.5)$. The median needling rate was $33 \%$.

The mean (SD) and median numbers of needling procedures per eye at 24 months were $1.6(1.1)$ and 1.0 in the overall population, with a range of 1 to 6 . The majority of needled eyes $(n=56 / 83 ; 67.5 \%)$ had one procedure (Table 4), and the mean time (SD) to the first needling was 152 (160) days (median, 90 days). In 74/83 (89.2\%) cases, an antifibrotic agent (MMC, 5-FU, or other) was used during the procedure. The clinical success rate in needled eyes, as defined in the "Methods" section, was $59.2 \%$ at 12 months and $44.6 \%$ at 24 months.

\section{Safety}

Intraoperative complications were reported in $10(4.6 \%)$ eyes, the most common being anterior chamber bleeding in $6(2.8 \%)$ eyes (Table 5). A total of 65/218 (29.8\%) eyes had one or more postoperative ocular AEs (Table 6); glaucoma-related SSI due to uncontrolled IOP $(n=14$ / $218 ; 6.4 \%$ - the most common being trabeculectomy) and hyphema $(n=10 / 218 ; 4.6 \%)$ were most frequently reported (Table 6). Overall, 44/218 (20.2\%) eyes had numeric hypotony $(<6 \mathrm{mmHg}$, self-resolved) within the first 2 postoperative weeks. Only $5 / 218$, however, were recorded as AEs, all of which occurred within 1 week postimplantation and resolved by the 1-month visit without any intervention. No eyes had persistent hypotony, defined in the protocol as IOP $<6 \mathrm{mmHg}$ at two consecutive postoperative visits $>30$ days apart. Notably, there were no clinical hypotony-related complications (such as flat anterior chamber with iridocorneal touch extending to the pupil, hypotony maculopathy, and choroidal effusion lasting $>30$ days [requiring surgical intervention]), retinal detachment, vitreous hemorrhage, or any other AE causing permanent visual impairment.

Three patients died during the study (due to decompensated cirrhosis, cardiac arrest, and hepatocellular carcinoma-not device/procedure-related), one of whom had both eyes implanted. Twelve patients (14 eyes) experienced non-fatal serious AEs (SAEs), of whom seven (nine eyes) had systemic SAEs. Six patients (six eyes) had ocular SAEs, five of which were in study eyes: 
a

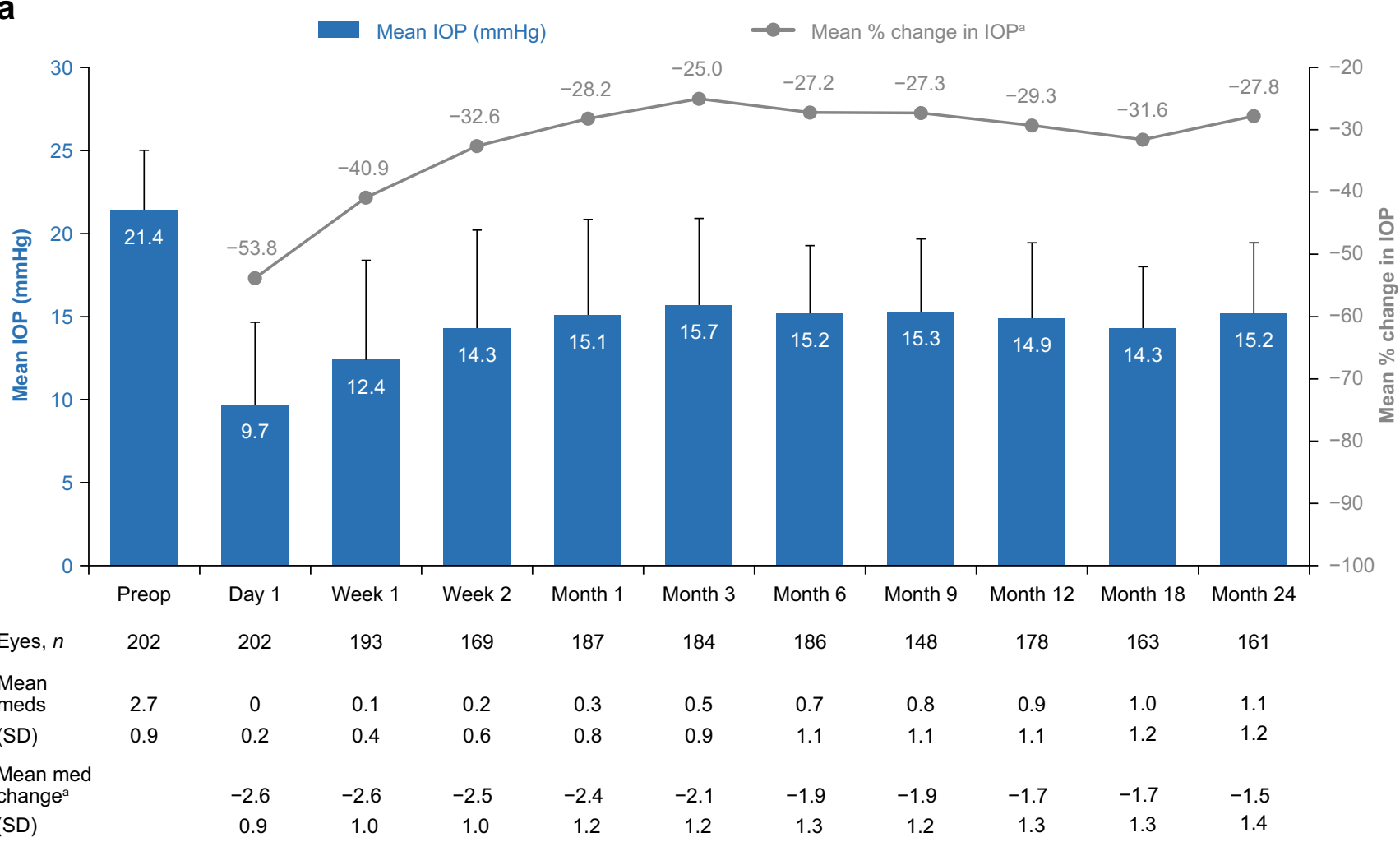

\section{b}

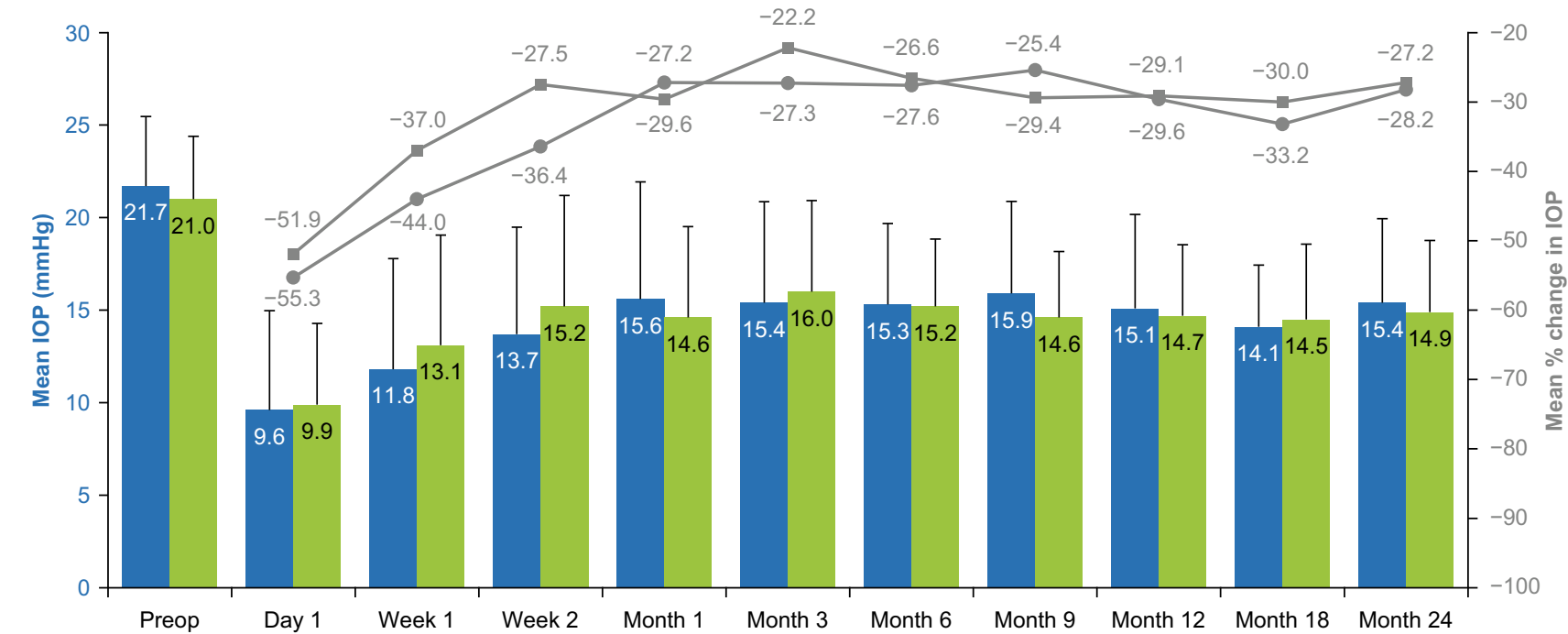

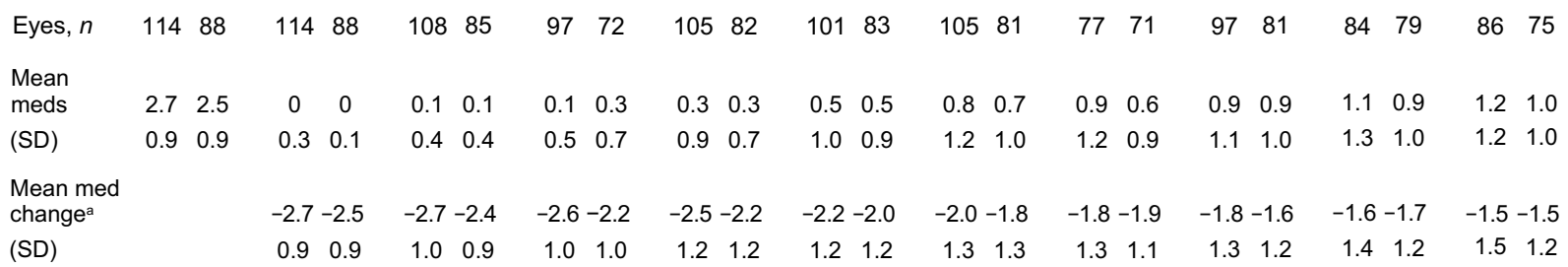

Fig. 2 Mean and changes in mean IOP and number of IOP-lowering medications from preoperative baseline over time in the mITT population (a) and implant alone vs. phaco + implant groups (b). IOP, intraocular pressure; meds, medications; mITT, modified intent-to-treat; phaco, phacoemulsification with intraocular lens replacement; preop, preoperative; $\mathrm{SD}$, standard deviation. ${ }^{\mathrm{a}} P<0.001$ at all postoperative visits 


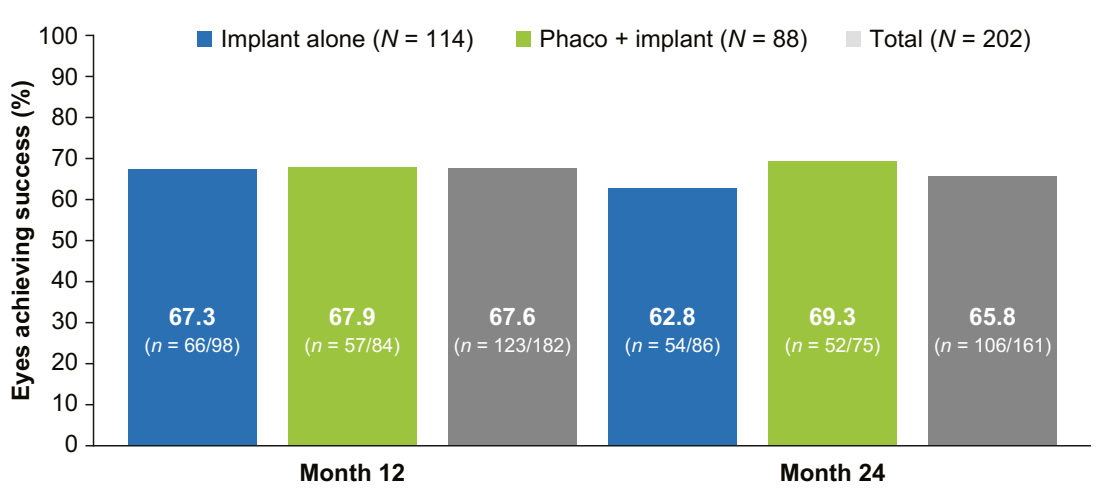

Fig. 3 Percentage of eyes with available data achieving success in the mITT population at months 12 and 24. Success was defined by eyes achieving $\geq$ $20 \%$ IOP reduction from baseline on the same or fewer medications without

cataract aggravated, retinal disorder (central retinal vein occlusion reported at 12 months, without elevated IOP), conjunctival erosion (implant exposure), glaucoma-related SSI (due to hospitalization for surgery), endophthalmitis (reported 15 months after implantation-detail provided in Table 6), and high IOP with SSI (cyclodestructive procedure) in the untreated fellow eye ( $n=1$ each; Table 6$)$. One patient had both systemic and ocular SAEs.

Not unexpectedly, mean best-corrected visual acuity (SD, $\log$ MAR) improvements from baseline at 12 and 24 months were noted in the phaco + implant group $(-0.27$ [0.24] and $-0.23[0.24])$, compared with the implant alone group $(-0.02$ [0.19] and 0.01 [0.21]), respectively. Changes in mean central corneal thickness (SD) were not statistically significant at 12 and 24 months. The change from baseline in average visual field mean deviation was not statistically significant at 24 months $(-1.0[8.3] ; P=0.138)$. glaucoma-related secondary surgical intervention (SSI) or intention to be converted to another procedure. mITT, modified intent-to-treat; phaco, phacoemulsification with intraocular lens replacement

\section{Discussion}

This prospective, 24-month, non-randomized, open-label, multicenter study conducted in typical clinical settings assessed the long-term effectiveness and safety of the gelatin implant in patients with POAG uncontrolled on topical IOPlowering medications. Mean IOP was reduced from 21.4 (3.6) (medicated baseline) to $14.9(4.5) \mathrm{mmHg}$ at month 12 and 15.2 (4.2) $\mathrm{mmHg}$ at month 24; the mean IOP-lowering medication count decreased from $2.7(0.9)$ at baseline to 0.9 (1.1) at month 12 and 1.1 (1.2) at month 24. Similar results were observed in both treatment groups at all postoperative visits up to 24 months $(P>0.4$, between-group comparisons). In addition, no differences in outcomes were noted at 24 months in pseudophakic eyes that received the implant alone, compared with the overall implant alone group and the phaco + implant group. These findings are consistent with other reports of

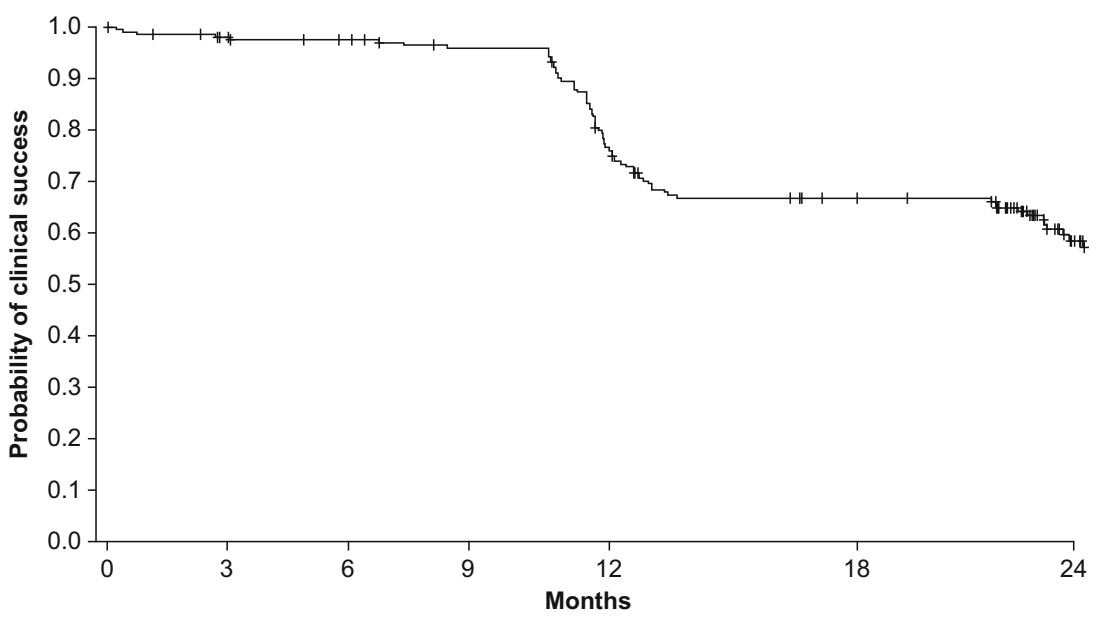

Fig. 4 Kaplan-Meier curve showing the probability of achieving success criteria at months 12 and 24 (mITT population). Failures were due to glaucoma-related secondary surgical intervention (SSI-which did not include needling) during the study period, or IOP reduction $<20 \%$ on the same number of medications or fewer at 12 and 24 months. Right censoring assumption was used. Probability of success was $67 \%$ at month
12 and $58 \%$ at month 24 . Note: eyes that did not undergo SSI but failed to meet success criteria at month 12 were deemed to have failed, even if they met the success criteria at month 24 . Tick signs indicate censored times, corresponding to incidences when eyes discontinued early or completed the study without experiencing any failures. IOP, intraocular pressure; mITT, modified intent-to-treat 
Fig. 5 Scatter plots of IOP reduction as a function of preoperative IOP at month 12 (a) and month 24 (b) in the mITT population. Each data point represents one eye. The gray line marks the lower limit of medicated IOP required at baseline for eligibility $(18 \mathrm{mmHg})$. The blue line delineates IOP reduction (lower portion) from IOP increase (upper portion), relative to baseline IOP. Data points below the 20,30, and $40 \%$ IOP reduction lines achieved that level of IOP lowering or more. IOP, intraocular pressure; mITT, modified intent-to-treat. ${ }^{\text {a }}$ Indicates IOP reduction success, as defined in the protocol
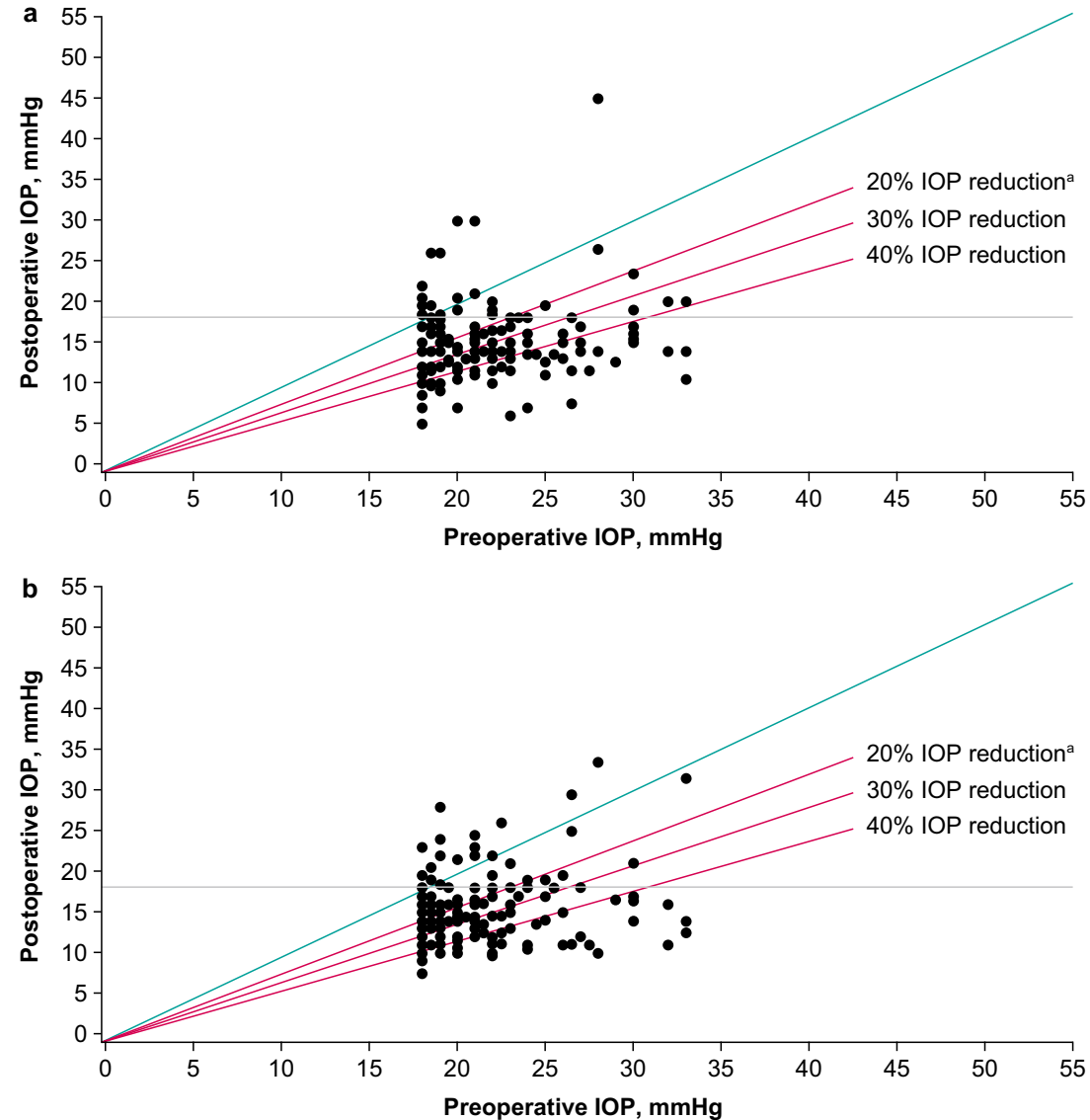

studies with this device, including the US pivotal trial in refractory glaucoma [23], as well as independent, retrospective $[14,17,18,24-26]$ and prospective $[12,13,15,16,19$, 27-29] studies in glaucoma, showing effectiveness at 1 year. Among those, a prospective, open-label study of the implant used alone or in combination with cataract surgery $(N=149$ eyes) [16] showed that the mean medicated IOP and mean number of medications decreased from 20.0 (7.1) $\mathrm{mmHg}$ and $1.9(1.3)$ at baseline to $13.9(4.3) \mathrm{mmHg}(P<0.01)$ and $0.5(0.8)(P<0.001)$ at 1 year, respectively. In our study, the mean percentage change in IOP from medicated baseline was $-29.3 \%$ at month 12 , consistent with those published by Mansouri et al. (31\% reduction) [16] and Grover et al. (35.6\% reduction) [23], for example.

Although the patient populations and mode of administration of adjunctive antifibrotic therapy differed in the study by Grover et al. [23], the one by Mansouri et al. [16], and ours, the effectiveness of the gel stent in reducing IOP and need for IOP-lowering medications appear similar. In addition, our results not only demonstrate continued effectiveness of the gelatin implant at 2 years, with a mean \% IOP reduction of $27.8 \%$, but also show strikingly stable IOP values from month 1 to 2 years (despite a small, expected elevation at month 3 that may correlate with the median time to first needling). The clinical success rate also remained stable between months 12
(67.6\%) and $24(65.8 \%)$, further supporting the long-term effectiveness of the gelatin implant. Overall, 60.7 and $62.7 \%$ had IOP $\leq 15 \mathrm{mmHg}$ at 12 and 24 months, respectively. It is also notable that the results were comparable whether implantation was performed as a stand-alone procedure or in combination with cataract surgery.

Needling can be an effective intervention in the postoperative management of gelatin stent implantation to restore bleb function, in line with recommendations by the American Academy of Ophthalmology after trabeculectomy [1]. There was variation in needling rate between study sites, as evidenced by the difference between the overall needling rate and the median needling rate. Overall, $41.1 \%$ of eyes underwent at least one needling procedure $(74.7 \%[n=62 /$ 83 ] occurring within the first 6 months post-surgery), and $44.6 \%$ of the needled eyes achieved clinical success criteria at month 24 , with comparable results in both treatment groups.

The study results are also clinically relevant when compared with other MIGS devices. For instance, in a 2-year pivotal trial, no statistically significant difference in mean IOP reduction from a washed-out baseline was reported at 24 months between patients who received the trabecular micro-bypass stent during cataract surgery (mean IOP: 18.6 [3.4] $\mathrm{mmHg}$ at baseline, 17.1 [2.9] $\mathrm{mmHg}$ at 24 months) and 
a

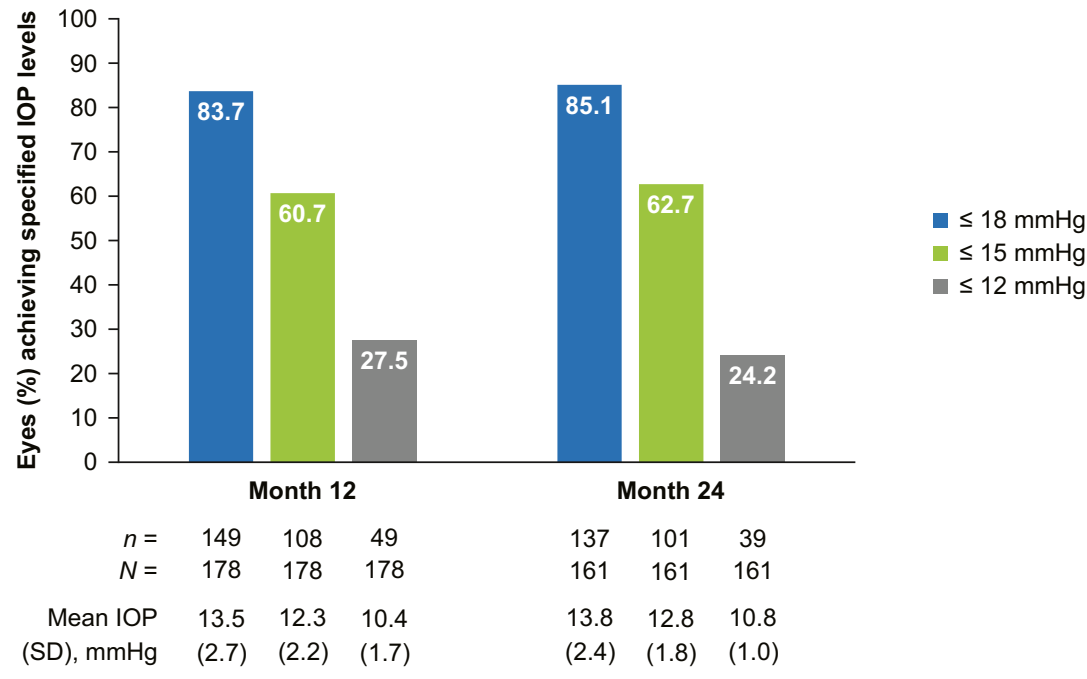

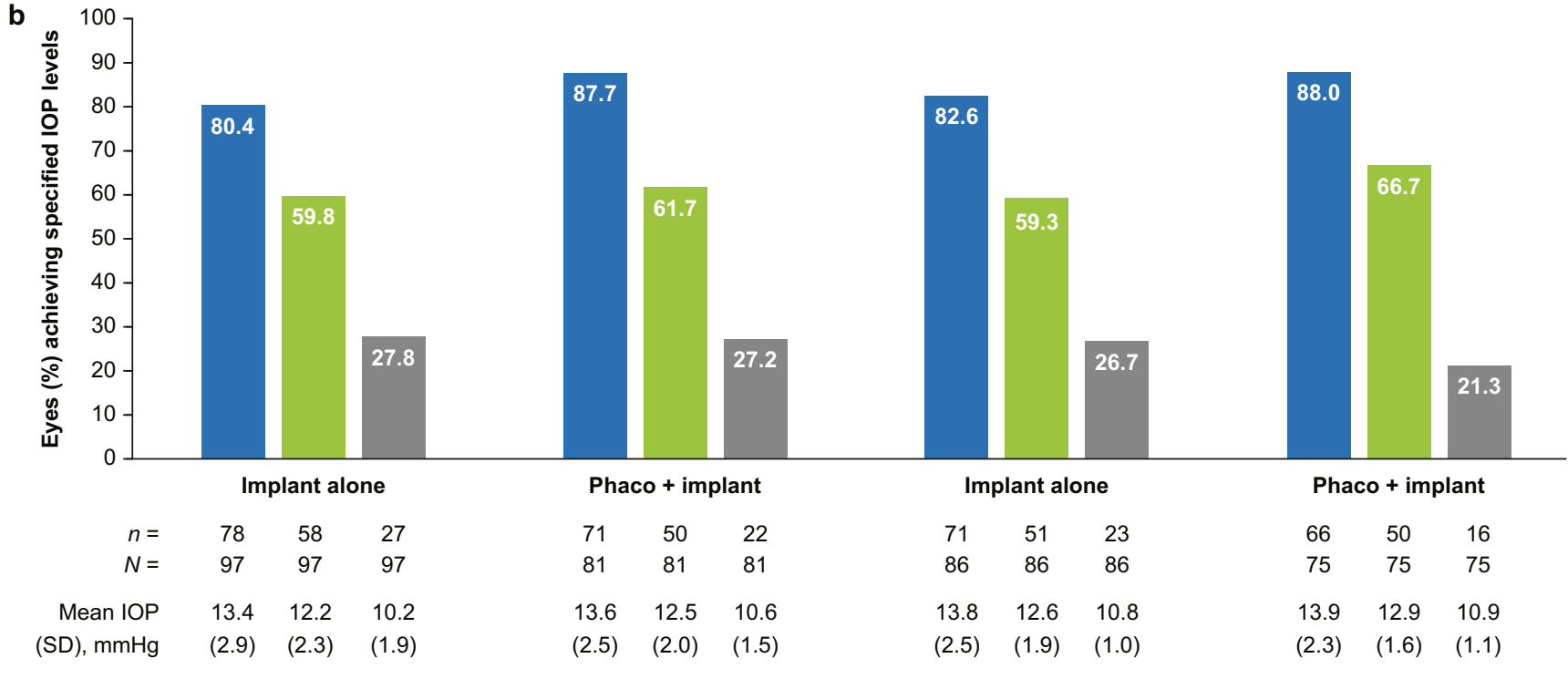

Month 12

Month 24

Fig. 6 Number and percentage of eyes achieving specified IOP levels in the overall mITT population (a) and implant alone vs. phaco + implant groups (b). IOP, intraocular pressure; mITT, modified intent-to-treat; phaco, phacoemulsification with intraocular lens replacement; SD, standard deviation

those who underwent cataract surgery alone (mean IOP: 17.9 [3.0] $\mathrm{mmHg}$ at baseline, 17.8 [3.3] $\mathrm{mmHg}$ at 24 months) [30]. Similarly, the mean number of IOP-lowering medications used at 24 months was not statistically significantly different between treatment groups [30], suggesting limited long-term effectiveness of the device. We did not expect to see additional IOP lowering in the phaco + implant group, because many studies looking at trabeculectomy and phaco-trabeculectomy have shown comparable IOP lowering with both procedures [31-38]. Both phacoemulsification and trabeculectomy techniques have evolved, which might explain why more recent papers report no differences in outcomes between trabeculectomy alone vs combined with phacoemulsification. The gelatin stent relies on a similar outflow pathway as trabeculectomy [39-41], but is a much less invasive procedure and provides a more controlled outflow; these factors likely explain the lack of differences between the two groups observed in our study.

How the effectiveness of the trabecular micro-bypass stent compares with that of the gelatin implant remains to be determined because the primary and secondary outcomes in studies of the trabecular micro-bypass stent assessed IOP lowering from unmedicated/washed-out baseline [10, 30]. In our study, eyes did not undergo washout before surgery, so the baseline IOP was expectedly lower. Also, most patients included in this study had moderate POAG, with an average visual field mean deviation of $-8.0 \mathrm{~dB}$, compared with $-3.9 \mathrm{~dB}$ in the trabecular micro-bypass study $[10,30]$. 
Table 3 Mean IOP at months 12 and 24 in eyes that were IOP-lowering medication-free (mITT population)

\begin{tabular}{llll}
\hline Visit & \multicolumn{2}{l}{ Mean IOP, mmHg (SD) } \\
\cline { 2 - 4 } & $\begin{array}{l}\text { Implant alone } \\
N=114\end{array}$ & $\begin{array}{l}\text { Phaco + implant } \\
N=88\end{array}$ & $\begin{array}{l}\text { Total } \\
N=202\end{array}$ \\
\hline Month 12 & $13.8(3.4)$ & $13.7(2.7)$ & $13.8(3.1)$ \\
Baseline IOP & $21.9(3.5)$ & $20.6(3.5)$ & $21.3(3.5)$ \\
n (\%) & $50 / 97(51.5)$ & $41 / 81(50.6)$ & $91 / 178(51.1)$ \\
Month 24 & $14.4(3.4)$ & $14.3(2.7)$ & $14.3(3.1)$ \\
Baseline IOP & $22.1(4.0)$ & $20.6(3.6)$ & $21.4(3.9)$ \\
$n(\%)^{\mathrm{a}}$ & $39 / 86(45.3)$ & $33 / 75(44.0)$ & $72 / 161(44.7)$ \\
\hline
\end{tabular}

IOP, intraocular pressure; mITT, modified intent-to-treat; phaco, phacoemulsification with intraocular lens placement; SD, standard deviation

${ }^{a}$ Based on the number of eyes with data available at baseline and the indicated visit

In studies of trabeculectomy, the gold standard for filtering surgery in open-angle glaucoma, effective IOP lowering to low teens was reported, but this was associated with significant AEs. Although the Tube versus Trabeculectomy study did not report outcomes at 2 years, results at 1 [42] and 3 years [43] showed that 57 and $60 \%$ of patients in the trabeculectomy arm experienced postoperative complications, respectively, compared with $29.8 \%$ at 2 years in our study. In a retrospective study that evaluated the outcomes and risk factors for failure of the gelatin stent versus trabeculectomy [24], both procedures had a 75\% survival of approximately 10 months without medications or additional surgery (complete success) and $>2$ years with add-on medications or laser trabeculoplasty (qualified success). Notably, one quarter and one third of eyes treated with the gelatin stent and trabeculectomy, respectively, were receiving glaucoma medications at the last recorded visit [24].

In line with the increasing trend of subconjunctival injection of MMC in trabeculectomy, all eyes implanted in this study received subconjunctival antifibrotic injection (range: 10-80 $\mu \mathrm{g}$ for MMC; two patients received 500- $\mu \mathrm{g} 5-\mathrm{FU}$ ) to

Table 4 Number (\%) of eyes that underwent 1, 2, 3, or $>3$ needling procedures (mITT population) ${ }^{\mathrm{a}}$

\begin{tabular}{llll}
\hline $\begin{array}{l}\text { Number of needling } \\
\text { procedures up to } \\
\text { month } 24\end{array}$ & $\begin{array}{l}\text { Implant alone } \\
N=50 / 114\end{array}$ & $\begin{array}{l}\text { Phaco + implant } \\
N=33 / 88\end{array}$ & $\begin{array}{l}\text { Total } \\
N=83 / 202\end{array}$ \\
\hline 1 & $35(70.0)$ & $21(63.6)$ & $56(67.5)$ \\
2 & $8(16.0)$ & $6(18.2)$ & $14(16.9)$ \\
3 & $3(6.0)$ & $5(15.2)$ & $8(9.6)$ \\
$>3$ & $4(8.0)$ & $1(3.0)$ & $5(6.0)$ \\
\hline
\end{tabular}

mITT, modified intent-to-treat; phaco, phacoemulsification with intraocular lens placement

${ }^{a}$ Among study eyes that underwent needling at any time point
Table 5 Summary of intraoperative complications (safety population)

\begin{tabular}{llll}
\hline Intraoperative complications & $\begin{array}{l}\text { Implant alone } \\
N=120\end{array}$ & $\begin{array}{l}\text { Phaco }+ \\
\text { implant } \\
N=98\end{array}$ & $\begin{array}{l}\text { Total } \\
N=218\end{array}$ \\
\hline Total & $3(2.5)$ & $7(7.1)$ & $10(4.6)$ \\
Anterior chamber bleeding & $1(0.8)$ & $5(5.1)$ & $6(2.8)$ \\
$\begin{array}{l}\text { Iris damage } \\
\text { Subconjunctival hemorrhage }\end{array}$ & $1(0.8)$ & $1(1.0)$ & $1(0.5)$ \\
$\begin{array}{l}\text { Vitreous in the pupil plane } \\
\text { (aphakic eye) }\end{array}$ & $1(0.8)$ & 0 & $1(0.5)$ \\
$\begin{array}{l}\text { Zonular disinsertion } \\
\text { c }\end{array}$ & 0 & $1(1.0)$ & $1(0.5)$ \\
\hline
\end{tabular}

Phaco, phacoemulsification with intraocular lens placement

${ }^{\text {a }}$ Characterized as excessive in five eyes

${ }^{\mathrm{b}}$ Underwent vitrectomy

${ }^{\mathrm{c}}$ Phacodonesis had been recorded prior to the surgery

allow precise dosing, compared with the traditional sponge method $[44,45]$. The study thus adds to the prospective data on the perioperative administration of MMC by subconjunctival injection with implantation of the gelatin stent, at dosages aligned with expert recommendations (10$40 \mu \mathrm{g})[46]$.

The device exhibited an acceptable safety profile. All cases of hypotony (defined as IOP $<6 \mathrm{mmHg}$ ) were self-limited and self-resolved within 1 month of surgery, similar to what was reported by Grover et al. [23]. Low IOP in the immediate postimplantation period seems less likely to lead to clinical hypotony-related complications, compared with similar IOP after trabeculectomy, and thus may be amenable to observation without immediate intervention [8, 47]. Although SAEs were rare during the 2-year study, the isolated case of endophthalmitis underscores the need for ongoing care and monitoring of patients following glaucoma filtering procedures, even when IOP is well controlled post-surgery.

Potential study limitations include some variability in the perioperative regimens, which may have impacted the study outcomes. Current recommendations from surgeons experienced with the gelatin stent suggest that preoperative preparation of the conjunctiva and ocular surface, placement closer to the 12 o'clock position, avoiding penetration of Schlemm's canal during implantation, making sure that the implant is free and mobile under the conjunctiva at the end of surgery, and achieving specific target IOP on day 1 or a low week-1 delta IOP, among others, may help optimize outcomes; most, however, were not published and thus not implemented during this study [46]. At the time of initiation of this study, the gelatin stent was very new on the market and no best practices were established, so the study results also reflect the investigators' learning curve with the surgery [46] and the variation in preand postoperative regimens associated with typical clinical settings. Another potential limitation is the fact that $<5 \%$ of the study population was of Asian and Black/African ethnicity 
Table 6 Ocular adverse events reported throughout the 2-year study (safety population)

\begin{tabular}{|c|c|}
\hline Ocular adverse events, $n(\%)$ & Total, $N=218$ eyes \\
\hline Secondary surgical intervention & $14(6.4)$ \\
\hline Trabeculectomy & $9(4.1)$ \\
\hline XEN@45 gelatin stent & $2(0.9)$ \\
\hline iStent ${ }^{\circledR}$ & $1(0.5)$ \\
\hline Ex-Press ${ }^{\circledR}$ shunt & $1(0.5)$ \\
\hline SLT & $1(0.5)$ \\
\hline Hyphema $^{\mathrm{a}}$ & $10(4.6)$ \\
\hline Device fracture & $6(2.8)$ \\
\hline IOP increase & $6(2.8)$ \\
\hline Study eye & $5(2.3)$ \\
\hline Fellow eye & $1(0.5)$ \\
\hline Hypotony $^{\mathrm{b}}$ & $5(2.3)$ \\
\hline YAG capsulotomy & $5(2.3)$ \\
\hline Choroidal effusion $^{\mathrm{c}}$ & $4(1.8)$ \\
\hline Conjunctival erosion & $4(1.8)$ \\
\hline Implant blockage by iris & $3(1.4)$ \\
\hline Eye pain & $3(1.4)$ \\
\hline Blepharitis & $2(0.9)$ \\
\hline Drug allergy & $2(0.9)$ \\
\hline Dysesthesia & $2(0.9)$ \\
\hline Iritis & $2(0.9)$ \\
\hline Retinal disorder ${ }^{\mathrm{d}}$ & $2(0.9)$ \\
\hline Shallow anterior chamber & $2(0.9)$ \\
\hline Subconjunctival hemorrhage & $2(0.9)$ \\
\hline Cataract aggravated & $1(0.5)$ \\
\hline Conjunctivitis & $1(0.5)$ \\
\hline Corneal epithelium defect & $1(0.5)$ \\
\hline Corneal infiltrates & $1(0.5)$ \\
\hline Device migration & $1(0.5)$ \\
\hline Endophthalmitis ${ }^{\mathrm{e}}$ & $1(0.5)$ \\
\hline Iris injury & $1(0.5)$ \\
\hline Keratitis, bacterial & $1(0.5)$ \\
\hline Macular edema & $1(0.5)$ \\
\hline Uveitic glaucoma $^{\mathrm{f}}$ & $1(0.5)$ \\
\hline Visual field progression & $1(0.5)$ \\
\hline
\end{tabular}

IOP, intraocular pressure; MMC, mitomycin C; SLT, selective laser trabeculoplasty; YAG, yttrium-aluminum-garnet

${ }^{a}$ Self-resolved, lasting $<30$ days

${ }^{\mathrm{b}}$ Defined as IOP $<6 \mathrm{mmHg}$ present at two consecutive postoperative visits $>30$ days apart

${ }^{\mathrm{c}}$ Self-limiting, lasting $>30$ days

${ }^{\mathrm{d}}$ Included CRVO (central retinal vein occlusion) with retinal hemorrhages and macular edema (IOP $14 \mathrm{mmHg}$ ), a serious AE reported at 24 months $(n=1)$, and vitrectomy (IOP $16 \mathrm{mmHg}$; not further detailed) reported at 18 months $(n=1)$

${ }^{\mathrm{e}}$ Endophthalmitis was reported 15 months after implantation and treated successfully with anterior chamber wash, vitrectomy, and intravitreal antibiotics. No implant erosion or blebitis was documented on any visits

${ }^{\mathrm{f}}$ Same eye that had endophthalmitis

(reported to have a higher risk of failure with trabeculectomy) [48-50]. Nevertheless, the results of this study demonstrate a favorable risk/benefit profile when compared with those published for more invasive surgeries like tube/trabeculectomy. Our findings are generalizable to eyes with POAG uncontrolled with topical hypotensive agents and provide evidence that can help clinical decision making.
As first surgical intervention, the gelatin implant was effective over 2 years in reducing both IOP and medication needs in patients with moderate POAG uncontrolled topically, with an acceptable safety profile. Used alone or in combination with cataract surgery, the gelatin implant lends itself to use earlier in the treatment paradigm, offering a minimally invasive surgical alternative for patients with target IOP in the mid-low teens who are uncontrolled on topical therapy or whose quality of life is low on topical polytherapy, as well as those who are non-adherent or intolerant to topical therapy.

Acknowledgments The authors would like to recognize the contributions of Zhanying Bai, M.S. and Charlie Wu, M.S. (employees of Allergan) for their support with the statistical analyses and programming, Andrew Shirlaw, M.S. (employee of Allergan) for his contribution to study project management, as well as Mini Balaram, M.D. (employee of Allergan) for her contribution to the manuscript development.

Writing/editorial assistance was provided to the authors by Michele Jacob, Ph.D., CMPP, of Evidence Scientific Solutions, Inc. (Philadelphia, Pennsylvania) and funded by Allergan plc (Dublin, Ireland). All authors met the ICMJE authorship criteria. Neither honoraria nor payments were made for authorship.

APEX Study Group Ejaz Ansari, M.D. (Maidstone Hospital, Eye, Ear, and Mouth Unit, Maidstone Kent, England). Leon Au, M.D. (Department of Eye Research, Manchester Royal Eye Hospital, Manchester, England). Keith Barton, M.D., CP FCRS, FRCOphth (Moorfields Eye Hospital, London, England). H. Burkhard Dick, M.D., Ph.D., FEBOS-CR (University Eye Clinic Bochum, Bochum, Germany). Luis Cadarso, M.D. (Ophthalmology Department, Clínica Oftalmológica Dr. Cadarso, Pontevedra, Spain). Antonio Fea, M.D. (Instituto di Fisiopatologia Clinica, Clinica Oculistica, Universita' di Torino, Torino, Italy). Fritz Hengerer, M.D. (Klinik für Augenheilkunde, Frankfurt, Germany). Helmut Höh, M.D., FEBO (Department of Ophthalmology, DietrichBonhoeffer-Klinikum, Neubrandenburg, Germany). Cosme LavinDapena, M.D. (Hospital Universitario La Paz, Madrid, Spain). Kin Sheng Lim, MBChB, M.D., FRCOphth (Ophthalmology Department, St Thomas' Hospital, London, England). Giorgio Marchini, M.D. (University Eye Clinic, Department of Neurological and Movement Sciences, University of Verona, Verona, Italy). Imran Masood, M.D. (Birmingham Midland Eye Theaters, West Midlands, England). Georg Mossböck, M.D. (Medical University Graz, Graz, Austria). Madhu Nagar, M.D. (Clinical Research Team, Pinderfields Hospital, Wakefield, England). Marco Nardi, M.D. (University of Pisa, Pisa, Italy). Herbert Reitsamer, M.D. (Department of Ophthalmology and Optometry, University Clinic Salzburg, SALK/Paracelsus Medical University, Salzburg, Austria). Marek Rękas, M.D., Ph.D. (Ophthalmology Department of the Military Health Service Institute, Warsaw, Poland). Tarek Shaarawy, M.D. (University of Geneva, Geneva, Switzerland). Ingeborg Stalmans, M.D., Ph.D. (Department of Ophthalmology, University Hospitals UZ Leuven, Leuven, Belgium). Miguel Teus, M.D. (Hospital Universitario Principe de Asturias, Madrid, Spain). Clemens Vass, M.D. (Vienna University, Vienna, Austria).

Funding information This study was funded by Allergan plc (Dublin, Ireland; formerly AqueSys, Inc.).

\section{Compliance with ethical standards}

Conflict of interest Herbert Reitsamer has received consulting honoraria from Allergan. Chelvin Sng has received consulting honoraria from Allergan, Glaukos (San Clemente, California), Alcon (Fort Worth, 
Texas), and Santen Pharmaceuticals (Osaka, Japan). Vanessa Vera has received consulting honoraria from Allergan. Keith Barton has received consulting honoraria from Alcon, Allergan, and Glaukos. Ingeborg Stalmans has received consulting honoraria from Allergan. Markus Lenzhofer declares that he has no conflict of interest.

Ethical approval All procedures performed in studies involving human participants were in accordance with the ethical standards of the institutional research committee and with the 1964 Helsinki declaration and its later amendments or comparable ethical standards.

Informed consent Informed consent was obtained from all individual participants included in the study.

Open Access This article is distributed under the terms of the Creative Commons Attribution 4.0 International License (http:// creativecommons.org/licenses/by/4.0/), which permits unrestricted use, distribution, and reproduction in any medium, provided you give appropriate credit to the original author(s) and the source, provide a link to the Creative Commons license, and indicate if changes were made.

Publisher's Note Springer Nature remains neutral with regard to jurisdictional claims in published maps and institutional affiliations.

\section{References}

1. American Academy of Ophthalmology (2015) Primary open-angle glaucoma - preferred practice pattern. http://www.aaojournal.org/ article/S0161-6420(15)01276-2/pdf. Accessed January 23, 2018

2. European Glaucoma Society Terminology and guidelines for glaucoma (4th edition). https://www.eugs.org/eng/guidelines.asp. Accessed January 23, 2018

3. Lichter PR, Musch DC, Gillespie BW, Guire KE, Janz NK, Wren PA, Mills RP, CIGTS Study Group (2001) Interim clinical outcomes in the Collaborative Initial Glaucoma Treatment Study comparing initial treatment randomized to medications or surgery. Ophthalmology 108:1943-1953. https://doi.org/10.1016/S01616420(01)00873-9

4. Feiner L, Piltz-Seymour JR (2003) Collaborative Initial Glaucoma Treatment Study: a summary of results to date. Curr Opin Ophthalmol 14:106-111

5. Jampel HD, Musch DC, Gillespie BW, Lichter PR, Wright MM, Guire KE (2005) Perioperative complications of trabeculectomy in the collaborative initial glaucoma treatment study (CIGTS). Am J Ophthalmol 140:16-22. https://doi.org/10.1016/j.ajo.2005.02.013

6. Zahid S, Musch DC, Niziol LM, Lichter PR (2013) Risk of endophthalmitis and other long-term complications of trabeculectomy in the Collaborative Initial Glaucoma Treatment Study (CIGTS). Am J Ophthalmol 155:674-680.e1. https://doi.org/10.1016/j.ajo.2012. 10.017

7. Gedde SJ, Feuer WJ, Shi W, Lim KS, Barton K, Goyal S, Ahmed IIK, Brandt J (2018) Treatment outcomes in the primary tube versus trabeculectomy study after 1 year of follow-up. Ophthalmology 125:650-663. https://doi.org/10.1016/j.ophtha.2018.02.003

8. Vera VI, Horvath C (2014) XEN gel stent: the solution designed by AqueSys ${ }^{\circledR}$. In: Samples JR, Ahmed IIK (eds) Surgical innovations in glaucoma. Springer Science+Business Media, New York, pp 189-198

9. Samuelson TW, Katz LJ, Wells JM, Duh YJ, Giamporcaro JE (2011) Randomized evaluation of the trabecular micro-bypass stent with phacoemulsification in patients with glaucoma and cataract.
Ophthalmology 118:459-467. https://doi.org/10.1016/j.ophtha. 2010.07.007

10. Vold S, Ahmed IIK, Craven ER, Mattox C, Stamper R, Packer M, Brown RH, Ianchulev T (2016) Two-year COMPASS trial results: supraciliary microstenting with phacoemulsification in patients with open-angle glaucoma and cataracts. Ophthalmology 123: 2103-2112. https://doi.org/10.1016/j.ophtha.2016.06.032

11. Caprioli J, Kim JH, Friedman DS, Kiang T, Moster MR, Parrish RK 2nd, Rorer EM, Samuelson T, Tarver ME, Singh K, Eydelman MB (2015) Special commentary: supporting innovation for safe and effective minimally invasive glaucoma surgery: summary of a joint meeting of the American Glaucoma Society and the Food and Drug Administration, Washington, DC, February 26, 2014. Ophthalmology 122:1795-1801. https://doi.org/10.1016/j.ophtha. 2015.02.029

12. De Gregorio A, Pedrotti E, Russo L, Morselli S (2017) Minimally invasive combined glaucoma and cataract surgery: clinical results of the smallest ab interno gel stent. Int Ophthalmol 38:1129-1134. https://doi.org/10.1007/s10792-017-0571-x

13. Galal A, Bilgic A, Eltanamly R, Osman A (2017) XEN glaucoma implant with mitomycin C 1-year follow-up: result and complications. J Ophthalmol 2017:5457246. https://doi.org/10.1155/2017/ 5457246

14. Hengerer FH, Kohnen T, Mueller M, Conrad-Hengerer I (2017) Ab interno gel implant for the treatment of glaucoma patients with or without prior glaucoma surgery: 1-year results. J Glaucoma 26: 1130-1136. https://doi.org/10.1097/ijg.0000000000000803

15. Sng CC, Wang J, Hau S, Htoon HM, Barton K (2017) XEN-45 collagen implant for the treatment of uveitic glaucoma. Clin Exp Ophthalmol 46:339-34. https://doi.org/10.1111/ceo.13087

16. Mansouri K, Guidotti J, Rao HL, Ouabas A, D'Alessandro E, Roy S, Mermoud A (2018) Prospective evaluation of standalone XEN gel implant and combined phacoemulsification-XEN Gel implant surgery: 1-year results. J Glaucoma 27:140-147. https://doi.org/10. 1097/IJG.0000000000000858

17. Tan SZ, Walkden A, Au L (2018) One-year result of XEN45 implant for glaucoma: efficacy, safety, and postoperative management. Eye 32:324-332. https://doi.org/10.1038/eye.2017.162

18. Ibáñez-Muñoz A, Soto-Biforcos VS, Chacón-González M, RúaGalisteo O, Arrieta-Los Santos A, Lizuain-Abadia ME, Del Río Mayor JL (2018) One-year follow-up of the XEN(R) implant with mitomycin-C in pseudoexfoliative glaucoma patients. Eur J Ophthalmol. https://doi.org/10.1177/1120672118795063

19. Mansouri K, Gillmann K, Rao HL, Guidotti J, Mermoud A (2018) Prospective evaluation of XEN gel implant in eyes with pseudoexfoliative glaucoma. J Glaucoma 27:869-873. https://doi. org/10.1097/ijg.0000000000001045

20. Parrish RK 2nd, Minckler DS, Lam D, Pfeiffer N, RojanaPongpun P (2009) Recommended methodology for glaucoma surgical trials. In: Shaarawy TM, Sherwood MB, Grehn F (eds) World Glaucoma Association Guidelines on design and reporting of glaucoma surgical trials. Kugler Publications, Amsterdam, pp 1-14

21. Armstrong RA (2013) Statistical guidelines for the analysis of data obtained from one or both eyes. Ophthalmic Physiol Opt 33:7-14. https://doi.org/10.1111/opo.12009

22. Rosner B, Glynn RJ, Lee ML (2003) Incorporation of clustering effects for the Wilcoxon rank sum test: a large-sample approach. Biometrics 59:1089-1098. https://doi.org/10.1111/j.0006-341X. 2003.00125.x

23. Grover DS, Flynn WJ, Bashford KP, Lewis RA, Duh YJ, Nangia RS, Niksch B (2017) Performance and safety of a new ab interno gelatin stent in refractory glaucoma at 12 months. Am J Ophthalmol 183:25-36. https://doi.org/10.1016/j.ajo.2017.07.023

24. Schlenker MB, Gulamhusein H, Conrad-Hengerer I, Somers A, Lenzhofer M, Stalmans I, Reitsamer H, Hengerer FH, Ahmed IIK (2017) Efficacy, safety, and risk factors for failure of standalone ab 
interno gelatin microstent implantation versus standalone trabeculectomy. Ophthalmology 124:1579-1588. https://doi.org/ 10.1016/j.ophtha.2017.05.004

25. Ozal SA, Kaplaner O, Basar BB, Guclu H, Ozal E (2017) An innovation in glaucoma surgery: XEN45 gel stent implantation. Arq Bras Oftalmol 80:382-385. https://doi.org/10.5935/00042749.20170093

26. Widder RA, Dietlein TS, Dinslage S, Kuhnrich P, Rennings C, Rossler G (2018) The XEN45 Gel Stent as a minimally invasive procedure in glaucoma surgery: success rates, risk profile, and rates of re-surgery after 261 surgeries. Graefes Arch Clin Exp Ophthalmol 256:765-771. https://doi.org/10.1007/s00417-0183899-7

27. Pérez-Torregrosa VT, Olate-Pérez A, Cerdà-Ibáñez M, GargalloBenedicto A, Osorio-Alayo V, Barreiro-Rego A, Duch-Samper A (2016) Combined phacoemulsification and XEN45 surgery from a temporal approach and 2 incisions. Arch Soc Esp Oftalmol 91:415421. https://doi.org/10.1016/j.oftal.2016.02.006

28. Fea AM, Spinetta R, Cannizzo PML, Consolandi G, Lavia C, Aragno V, Germinetti F, Rolle T (2017) Evaluation of bleb morphology and reduction in IOP and glaucoma medication following implantation of a novel gel stent. J Ophthalmol 2017:9364910. https://doi.org/10.1155/2017/9364910

29. Hohberger B, Welge-Lüßen UC, Lämmer R (2018) MIGS: therapeutic success of combined Xen Gel Stent implantation with cataract surgery. Graefes Arch Clin Exp Ophthalmol 256:621-625. https://doi.org/10.1007/s00417-017-3895-3

30. Craven ER, Katz LJ, Wells JM, Giamporcaro JE (2012) Cataract surgery with trabecular micro-bypass stent implantation in patients with mild-to-moderate open-angle glaucoma and cataract: two-year follow-up. J Cataract Refract Surg 38:1339-1345. https://doi.org/ 10.1016/j.jcrs.2012.03.025

31. Song BJ, Ramanathan M, Morales E, Law SK, Giaconi JA, Coleman AL, Caprioli J (2016) Trabeculectomy and combined phacoemulsification-trabeculectomy: outcomes and risk factors for failure in primary angle closure glaucoma. J Glaucoma 25: 763-769. https://doi.org/10.1097/ijg.0000000000000493

32. Jung LJ, Isida-Llerandi CG, Lazcano-Gomez G, SooHoo JR, Kahook MY (2014) Intraocular pressure control after trabeculectomy, phacotrabeculectomy and phacoemulsification in a Hispanic population. J Curr Glaucoma Pract 8:67-74. https:// doi.org/10.5005/jp-journals-10008-1164

33. Murthy SK, Damji KF, Pan Y, Hodge WG (2006) Trabeculectomy and phacotrabeculectomy, with mitomycin-C, show similar twoyear target IOP outcomes. Can J Ophthalmol 41:51-59. https:// doi.org/10.1016/s0008-4182(06)80067-0

34. Cillino S, Di Pace F, Casuccio A, Calvaruso L, Morreale D, Vadala M, Lodato G (2004) Deep sclerectomy versus punch trabeculectomy with or without phacoemulsification: a randomized clinical trial. J Glaucoma 13:500-506

35. Kuroda S, Mizoguchi T, Terauchi H, Nagata M (2001) Trabeculectomy combined with phacoemulsification and intraocular lens implantation. Semin Ophthalmol 16:168-171. https://doi. org/10.1076/soph.16.3.168.4203

36. Guggenbach M, Mojon DS, Böhnke M (1999) Evaluation of phacotrabeculectomy versus trabeculectomy alone. Ophthalmologica 213:367-370. https://doi.org/10.1159/ 000027456
37. Derick RJ, Evans J, Baker ND (1998) Combined phacoemulsification and trabeculectomy versus trabeculectomy alone: a comparison study using mitomycin-C. Ophthalmic Surg Lasers 29:707-713

38. Yu CB, Chong NH, Caesar RH, Boodhoo MG, Condon RW (1996) Long-term results of combined cataract and glaucoma surgery versus trabeculectomy alone in low-risk patients. J Cataract Refract Surg 22:352-357

39. Chang TC, Budenz DL, Liu A, Kim WI, Dang T, Li C, Iwach AG, Radhakrishnan S, Singh K (2012) Long-term effect of phacoemulsification on intraocular pressure using phakic fellow eye as control. J Cataract Refract Surg 38:866-870. https://doi. org/10.1016/j.jcrs.2012.01.016

40. Mansberger SL, Gordon MO, Jampel H, Bhorade A, Brandt JD, Wilson B, Kass MA (2012) Reduction in intraocular pressure after cataract extraction: the Ocular Hypertension Treatment Study. Ophthalmology 119:1826-1831. https://doi.org/10.1016/j.ophtha. 2012.02.050

41. Slabaugh MA, Bojikian KD, Moore DB, Chen PP (2014) The effect of phacoemulsification on intraocular pressure in medically controlled open-angle glaucoma patients. Am J Ophthalmol 157:2631. https://doi.org/10.1016/j.ajo.2013.08.023

42. Gedde SJ, Herndon LW, Brandt JD, Budenz DL, Feuer WJ, Schiffman JC (2007) Surgical complications in the Tube Versus Trabeculectomy Study during the first year of follow-up. Am J Ophthalmol 143:23-31. https://doi.org/10.1016/j.ajo.2006.07.022

43. Gedde SJ, Schiffman JC, Feuer WJ, Herndon LW, Brandt JD, Budenz DL (2009) Three-year follow-up of the Tube Versus Trabeculectomy Study. Am J Ophthalmol 148:670-684. https:// doi.org/10.1016/j.ajo.2009.06.018

44. Khouri SA, Huang G, Huang LY (2017) Intraoperative injection vs sponge-applied mitomycin $\mathrm{C}$ during trabeculectomy: one-year study. J Curr Glaucoma Pract 11:101-106. https://doi.org/10. 5005/jp-journals-10028-1233

45. Pakravan M, Esfandiari H, Yazdani S, Douzandeh A, Amouhashemi N, Yaseri M, Pakravan P (2017) Mitomycin Caugmented trabeculectomy: subtenon injection versus soaked sponges: a randomised clinical trial. Br J Ophthalmol 101:12751280. https://doi.org/10.1136/bjophthalmol-2016-309671

46. Vera V, Ahmed IIK, Stalmans I, Reitsamer H (2018) Gel stent implantation - recommendations for preoperative assessment, surgical technique, and postoperative management. US Ophthalmic Rev 11:38-46. https://doi.org/10.17925/USOR.2018.11.1.38

47. Vijaya L, Manish P, Ronnie G, Shantha B (2011) Management of complications in glaucoma surgery. Indian $\mathrm{J}$ Ophthalmol 59(Suppl):S131-S140. https://doi.org/10.4103/0301-4738.73689

48. Nguyen AH, Fatehi N, Romero P, Miraftabi A, Kim E, Morales E, Giaconi J, Coleman AL, Law SK, Caprioli J, Nouri-Mahdavi K (2018) Observational outcomes of initial trabeculectomy with mitomycin $\mathrm{c}$ in patients of African descent vs patients of European descent: five-year results. JAMA Ophthalmol 136:1106-1113. https://doi.org/10.1001/jamaophthalmol.2018.2897

49. Tan C, Chew PT, Lum WL, Chee C (1996) Trabeculectomysuccess rates in a Singapore hospital. Singap Med J 37:505-507

50. Wong JS, Yip L, Tan C, Chew P (1998) Trabeculectomy survival with and without intra-operative 5-fluorouracil application in an Asian population. Aust N Z J Ophthalmol 26:283-288. https:// doi.org/10.1111/j.1442-9071.1998.tb01331.x 